\title{
Transaction
}

\section{Molecular Dynamics Simulation for $\beta$-1,3-D-Glucan in Aqueous Solutions : Attachment of One Side Chain Induces an Entire Structural Change}

\author{
Kentaro Miyoshi ${ }^{* 1}$, Kazuya Uezu ${ }^{* 1}$, Kazuo Sakurai ${ }^{* 1}$, and Seiji Shinkai ${ }^{* 2}$ \\ ${ }^{* 1}$ Department of Chemical Processes and Environments, Faculty of Environmental Engineering, \\ The University of Kitakyushu, 1-1 Hibikino Wakamatsu-ku, Kitakyushu, Fukuoka 808-0135, Japan \\ ${ }^{* 2}$ Department of Chemistry and Biochemistry, Graduate School of Engineering, \\ Kyushu University, 744 Motooka Nishi-ku, Fukuoka 819-0395, Japan
}

\begin{abstract}
D-glucans form a right-handed 6 triple helix in water, and some of them have side chains, generally, consisting of $\beta-1,6$-glucose. Since the side chains can provide hydrophilicity to the molecule, the conformation and other physical properties can be drastically changed by introducing the side chain. To understand the side chain effect from the molecular level, we attached one $\beta-1,6$ side chain to a $\beta$-1,3-D-glucan main chain consisting of 19 D-glucose units, and carried out molecular dynamics (MD) simulations in water. The calculation showed that the attachment of the side chain induced the change of helical pitch, hydrogen bonding type, and the bending of the main chain for $\beta-1,3-\mathrm{D}-$ glucan structure. The water molecules neighboring the side chain were bound with hydrogen bonds rather tightly and the mobility was more reduced than that of other water molecules.
\end{abstract}

(Received 5 January, 2006 ; Accepted 14 July, 2006)

\section{Introduction}

The $\beta$-1,3-D-glucan family is one of the abundant polysaccharides on the earth.[1] In the last two decades, it has been found that $\beta-1,3$-D-glucans have useful biological activities, especially, anti-tumor[2] and antiAIDS virus[3] activities. These bio-activities make $\beta-1$, 3-D-glucans an attractive research topic in chemistry and biology. In nature, generally, $\beta-1,3-D-$ glucans form a right-handed triple helix consisting of the three glucan chains. [4-6] Some of the family, such as schizophyllan and lentinan, have a hydrophilic side chain. For example, Fig. 1 shows the chemical structure of schizophyllan.

The triple helix of $\beta$-1,3-D-glucans can be dissociated to three single chains by dissolving in dimethylsulfoxide (DMSO), and by exchanging DMSO for water, the single chains form aggregates.

Curdlan is the simplest member of the family consisting of only the $\beta-1,3-\mathrm{D}$-glucan main chain. Curdlan is insoluble in neutral water unless the molecular weight is low (ca. < 2000), because of absence of the hydrophilic side chains. The hydrophilic side chains make the glucans soluble in water, sometimes to produce a rather concentrated solution. Teramoto et al. [7] found an abnormal optical rotatory dispersion when the concentrated schizophyllan solution was cooled around the freezing point. They showed that this phenomenon is related to the cooperative motion of the schizophyllan side chain. The critical temperature to induce this motion strongly depends on the solvents, and even the deuteration of water causes a drastic increment of the temperature. They interpreted this phenomenon with a hypothesis that the motion of water molecules are more restricted around the side chains than that of the other waters including those bound to the main chain. Their

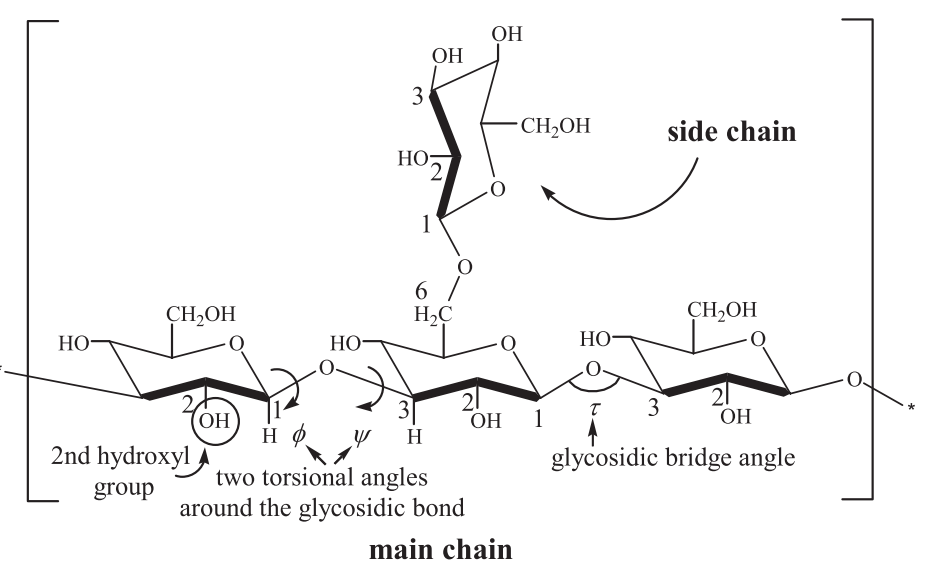

Fig. 1 Repeating unit of $\beta$-1,3-D-glucan (shizophyllan) and the definitions of the two torsional angles and the bridge angle. 
data indicate that the side chains of $\beta-1,3$-D-glucan play an important role in solution properties. Recently, we found that $\beta-1,3-D-$ glucans form a complex with some polynucleotides and the side chain glucose of the $\beta-1,3-\mathrm{D}-$ glucan affected thermal stabilization of this complex in aqueous solution; the stabilization of the complex was drastically increased by attaching only some side chains to the $\beta$-1,3-D-glucan main chain. [8] This phenomenon suggested that the small amount of the side chains could affect the structure of the $\beta-1,3-\mathrm{D}$-glucan.

This paper aims to clarify the structure of $\beta-1,3-\mathrm{D}-$ glucan in water in terms of molecular dynamics technique, focusing on how the existence of glucose side chain influences the entire helix and what kind of hydrogen bonds is dominant in aqueous $\beta-1,3-\mathrm{D}$-glucan. We examined two cases : one is curdlan triple helix with 19 glucoses on each chain, thus total 57 main chain glucoses are present and another is a hypothetical curdlan derivative in which only one main-chain glucose has an $\beta-1,6$ side chain. The side chain was attached to one of the 10th (right in the middle) glucose. For convenience, hereinafter, we denote the first and second models as CUR and $1 \mathrm{G}+\mathrm{CUR}$, respectively. We are interested in the structures in water; therefore, we filled the empty space in the calculation cell with water molecules.

\section{Results and discussion}

This section consists of three subsections. The first section describes how the presence of the glucose side chain affects the entire glucan structure, comparing CUR and $1 \mathrm{G}+\mathrm{CUR}$. Secondary, we will examine how this structural change is reflected to the hydrogen bonds array which maintains the triple helix. Finally, we will clarify a molecular origin for this phenomenon.

\section{2-1. Structures of CUR and $1 G+C U R$}

Unless we started the simulations from abnormal structures, the calculation seemed to reach a steady state within 100 ps from the fluctuation of internal energy. However, for curdlan model, the calculation might reach a complete steady state within 100-300ps from the fluctuation of the population of the hydrogen bonds. Fig. 2 compares the obtained CUR and $1 \mathrm{G}+\mathrm{CUR}$ structures. The CUR maintains the triple helix during the calculation and the helix is straight. Although the triple helix is still maintained in the $1 \mathrm{G}-\mathrm{CUR}$, the helix was bent centering on the attached side chain. Fig. 3 (a) presents the time fluctuation of averaged pitches for the two models, CUR and $1 \mathrm{G}+\mathrm{CUR}$ during 400ps. The pitches distribute centering around 20.6 and 18.2 for CUR and $1 \mathrm{G}+\mathrm{CUR}$ respectively: i.e., the pitch of $1 \mathrm{G}+\mathrm{CUR}$ is shorter than that of CUR and close to that of the curdlan crystal structure. This shortened pitch can be ascribed to the bending of the structure in $1 \mathrm{G}+\mathrm{CUR}$. Fig. 3(b) presents the position dependence of the pitch in $1 \mathrm{G}+\mathrm{CUR}$, showing that the glucose closer to the side chain has the shorter pitch. (a) CUR

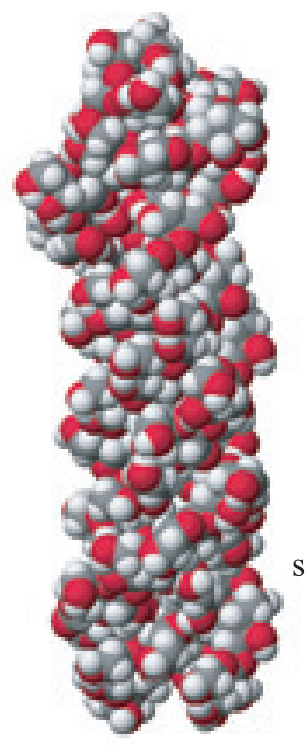

(b) $1 \mathrm{G}+\mathrm{CUR}$

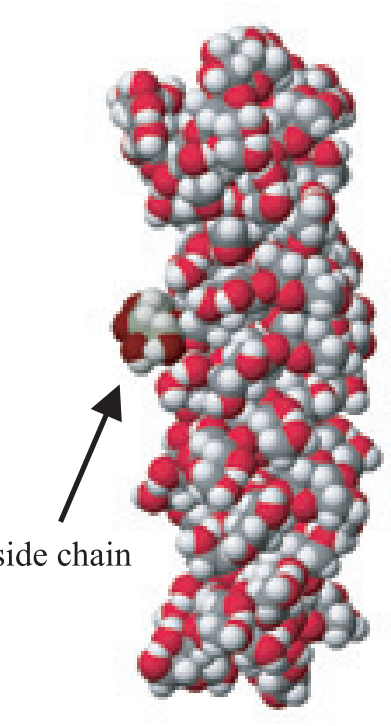

Fig. 2 The calculated structures with MD, comparing between (a) CUR and (b) 1G+CUR. Each single chain consists of 19 glucoses and $1 \mathrm{G}+$ CUR has a glucosyl side chain at the 10th main chain glucose with 1-6 linkage.

The calculation was carried out under the condition that water molecules are surrounding CUR or $1 \mathrm{G}+\mathrm{CUR}$. When we calculated the most stable structure of CUR without water at $0 \mathrm{~K}$, the pitch was $18 \AA$ that agrees with those obtained for the dehydrated curdlan. [4] Fig. 3, 4 indicates that the CUR main chain was stretched when immersed in water, however, only one side chain can pull back it to the dehydrated value. It is interesting that one side chain can provide such impact to the main-chain structure.

Fig. 5 shows the distribution of the three parameters $(\phi, \phi, \tau)$ for CUR and $1 \mathrm{G}+\mathrm{CUR}$. The bold lines indicate those for the curdlan crystal structure and the gray areas show the region from which the calculated model can take the values. Since $1 G+C U R$ is not much deformed from the crystal, $\phi$ and $\phi$ are similar to those of the crystal structure. Since we can consider the crystal values as the most stable ones (which is expected the structure at $0 \mathrm{~K}$ ), the rather wide gray region can be related to thermal fluctuation. For both models, $\tau$ is almost the same as that 


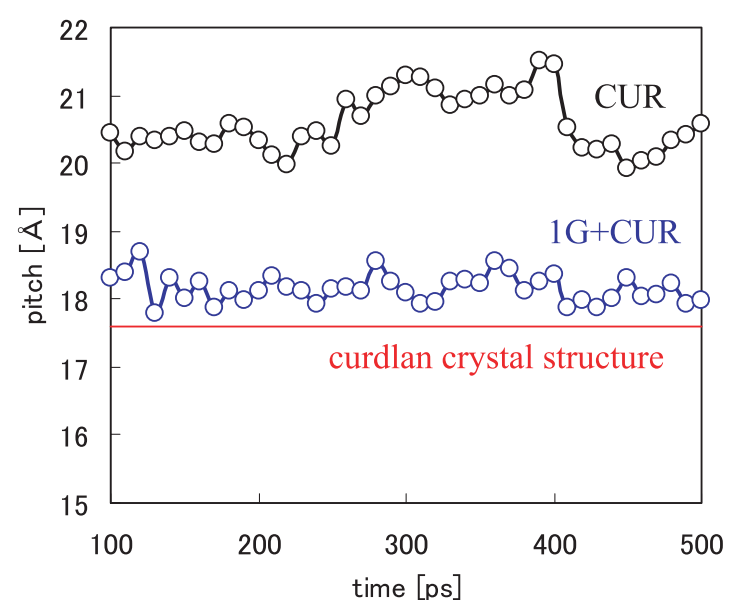

Fig. 3 Fluctuation of the helix pitches for CUR and 1G+

CUR models during 100-500ps. The red line indicates the pitch for a curdlan crystal structure determined by Deslandes et al. [6]

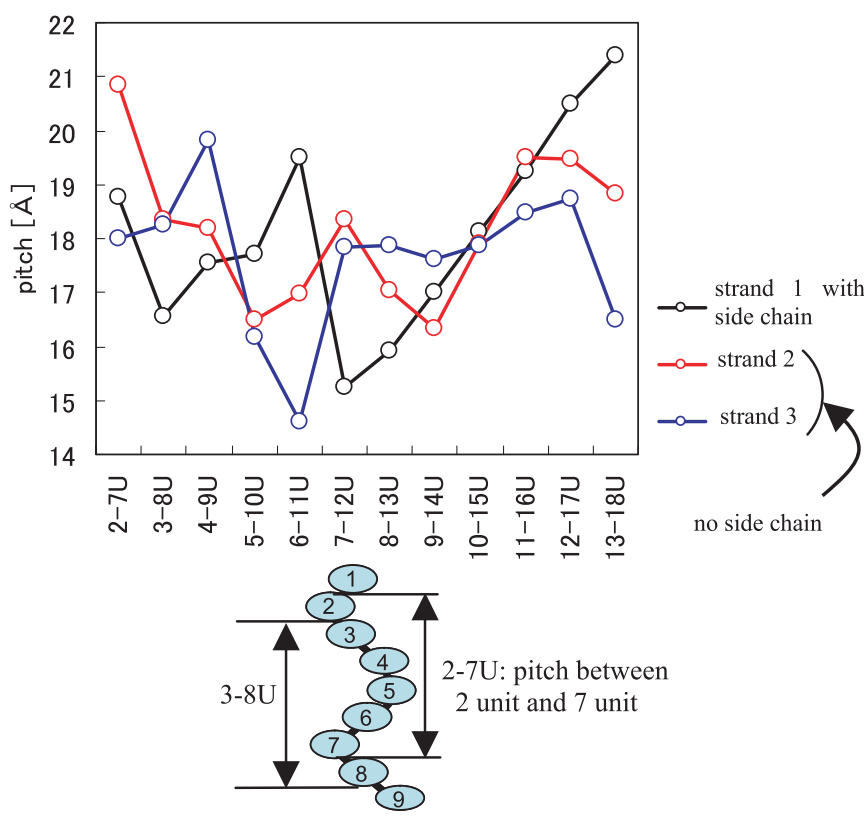

$6 / 1$ helix

Fig. 4 The position dependence of the helix pitch. The pitch is defined by the length between the 6 main chain because of the symmetry, thus we determined the position dependence of the helix pitch, for example, from the 2 nd to 7 th unit or 3 rd to 8th Unit, as presented by the under illustration.

of the crystal, indicating that it is difficult to bend the $\mathrm{C}-\mathrm{O}-\mathrm{C}$ angle between the main-chain glucoses. Fig. 5 demonstrates that the change in the pitch is mainly reflected to the rotating angle of $\phi$, splitting into two regions.

\section{2-2. Hydrogen Bonds in CUR and $1 G+C U R$}

There have been three possible structures proposed for the hydrogen bonds, as presented in Fig. 6, which (a) CUR
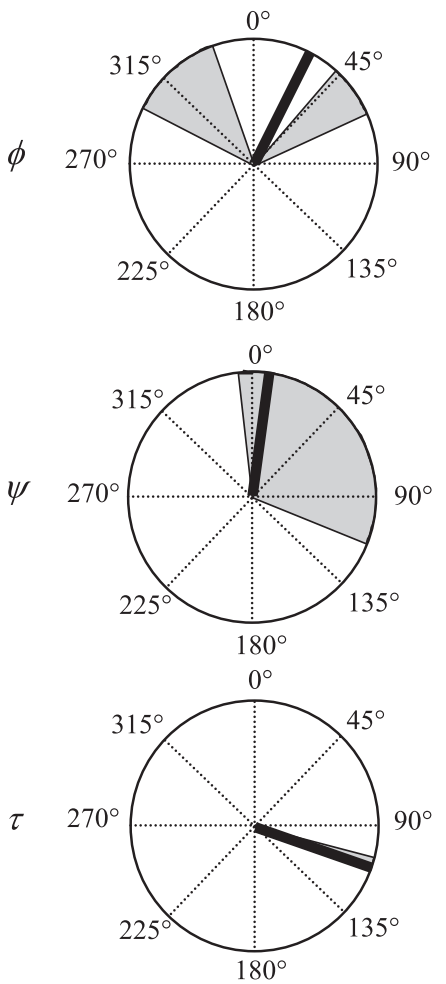

(b) $1 \mathrm{G}+\mathrm{CUR}$
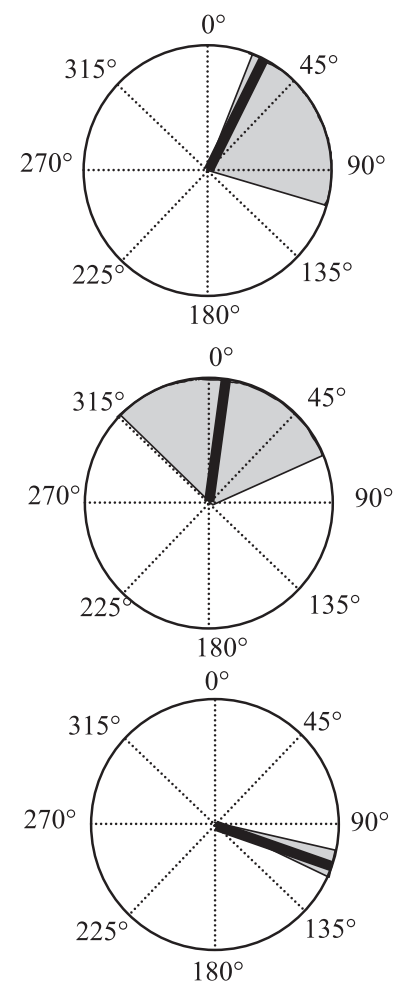

Fig. 5 Comparison of three conformational parameters (see Figure 1) between the MD calculation and the reported values for (a) CUR and (b) $1 \mathrm{G}+\mathrm{CUR}$. The thick black lines indicate the parameters of the curdlan crystal structure. The gray zones indicate observed values after calculation.

maintain the triple helix of $\beta-1,3-\mathrm{D}$-glucans. The type A denotes the hexagonal hydrogen bonds to form among the three glucans that locate at the same $x-y$ plane along the helix. Three 2nd hydroxyl groups (defined in Fig. 1) protrude toward the center of the helix and form the hexagonal shape and the hexagon is arranged perpendicular to the helix axis. This form was originally proposed for $\beta$-1,3-D-xylan by Atkins et al., [9] and subsequently extended to other $\beta-1,3$-D-glucans by Norisuye et al.[10] The type B is an intra-molecular hydrogen bond to form along the one $\beta$-1,3-D-glucan employing the adjacent $\mathrm{O} 2$ atoms on the same chain, and the helicity of this hydrogen bond array is the same as the glucan chain, i.e., right handed. This form was originally proposed by Sathyanarayana and Rao,[11] and is considered as a possible conformation in solutions. [12] The type $\mathrm{C}$ is an inter-molecular hydrogen bond which is constructed among the three $\mathrm{O} 2$ atoms in the different chains. As schematically presented in the figure, the hydrogen bond forms between the $\mathrm{O} 2$ atoms on different $\mathrm{x}-\mathrm{y}$ planes along the curdlan helix, hence the hydrogen bond is not perpendicular to the helix axis. The hydrogen 
(a) type A
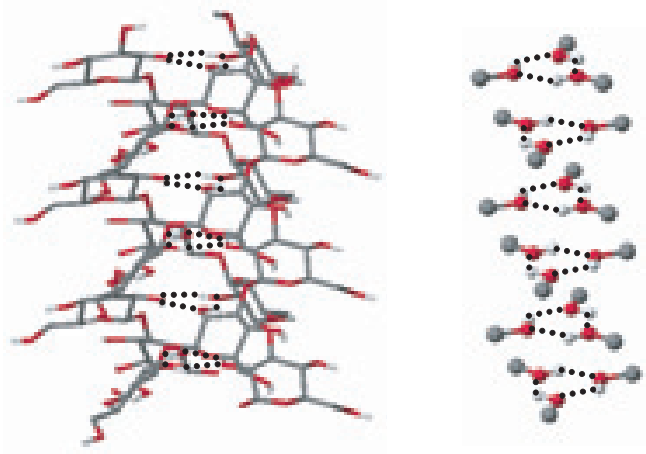

(b) type B
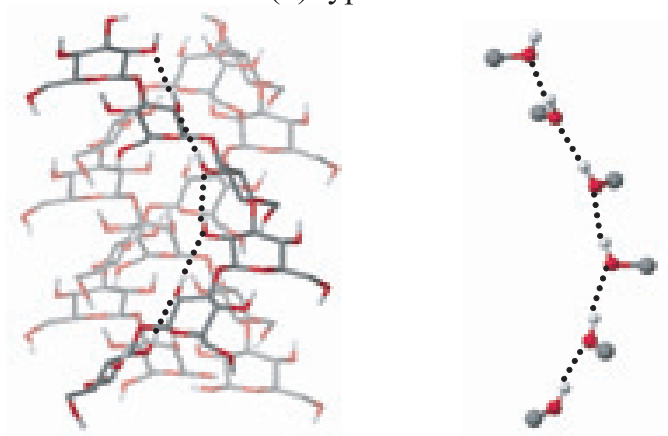

(c) type $\mathrm{C}$
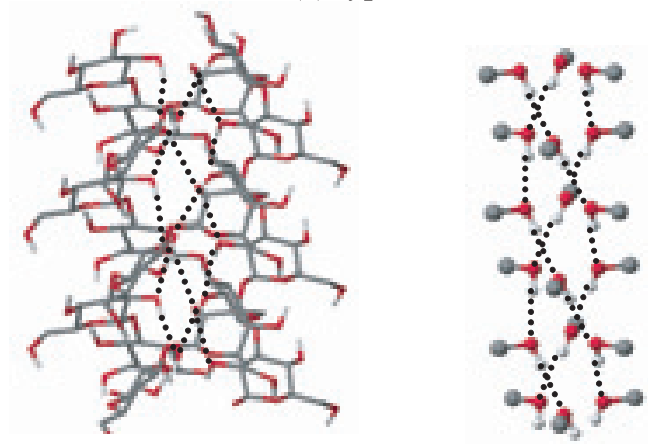

Fig. 6 Three possible structures proposed for the hydrogen bonds in the triple helix of $\beta-1,3-\mathrm{D}-$ glucans. Each model is presented by two styles; the overall feature of the triple helix (left) and 2nd $\mathrm{OH}$ and the hydrogen bonds abstracted from the helix. The dotted lines show the hydrogen bonds. (a) the type $\mathrm{A}$ is the hexagonal inter-molecular hydrogen bonds. The hydrogen bonds are formed between the different chains at the same $x-y$ plane. (b) the type $\mathrm{B}$ is the right handed helical intramolecular hydrogen bonds. The hydrogen bonds are formed between the adjacent $\mathrm{O} 2$ atoms in the same chain. (c) the type $\mathrm{C}$ is the left handed helical inter-molecular hydrogen bonding type. The hydrogen bonds are formed between different chains at the different $x-y$ plane.

bonds are connected along the helix, traversing three curdlan chains to make a left-handed helix, therefore, the hydrogen bond array makes a reverse helix of the main chain. Hydrogen bond was determined based on the hydrogen-donor distance and the angle made by covalent bonds to the donor and acceptor atoms (distance : less than $3.2 \AA$, angle : less than 120 degree).

At moderate temperatures, we can expect that three types of the hydrogen bonds emerge concurrently with a certain population proportional to the free energy difference. Fig. 7 shows how the population for each types of the hydrogen bonds is fluctuated, comparing with the fluctuation of the pitches for CUR (a) and $1 \mathrm{G}+\mathrm{CUR}$ (b). $1 \mathrm{G}+\mathrm{CUR}$ reached an equilibrium structure within 100 ps, while CUR took about 250-300 ps. This difference can be ascribed to a reason that we started the calculation from a dehydrated form which is closer to $1 \mathrm{G}+\mathrm{CUR}$ than to CUR. In CUR, the populations of types B and C were decreased in the range $280-400$ ps. At the exactly same period, the pitch was increased and the population of type $\mathrm{A}$ is also increased. The types B and C are formed in a longitudinal direction of the helix, therefore, the elongated pitch makes it difficult to form them. On the other hand, the horizontally formed type A should not be affected by the change in the pith length. In $1 G+C U R$, since the pitch is smaller than that of CUR, the dominant types are B and C. In terms of the heat of formation determined by MOPAC [13], type $\mathrm{C}$ is slightly more stable than type B. This energy difference may be reflected to the fact that the type $\mathrm{C}$ is the majority species for the most case.

\section{2-3. Water Molecules Surrounding the Side Chain of 1G+CUR}

The enlarged pitch in CUR was only observed when we filled the calculation cell with water molecules. This implies that water molecules should play an important role for this phenomenon. We carried out statistical analysis for the kinetics of water molecules around the side chain, using the mean square displacement $(M S D(t))$. $M S D(t)$ is the average distance that one molecule travels during the certain time, defined by the following equation :

$$
\operatorname{MSD}(t)=\left\langle\left|r_{i}(t)-r_{i}(0)\right|^{2}\right\rangle
$$

Here, $\left|\boldsymbol{r}_{i}(t)-\boldsymbol{r}_{i}(0)\right|$ is the distance traveling to by the $i$ th molecule over some time interval $t$. Fig. 8 plots $M S D(t)$ against $t$ during 8 ps for $1 \mathrm{G}+\mathrm{CUR}$, comparing between the water molecules around the side chain, those around the main chain and those in bulk. The slope of this plot can be related to the self-diffusion coefficient. As presented by the plots, the mobility for the water molecules near the side chain is considerably lower than 
(a) CUR

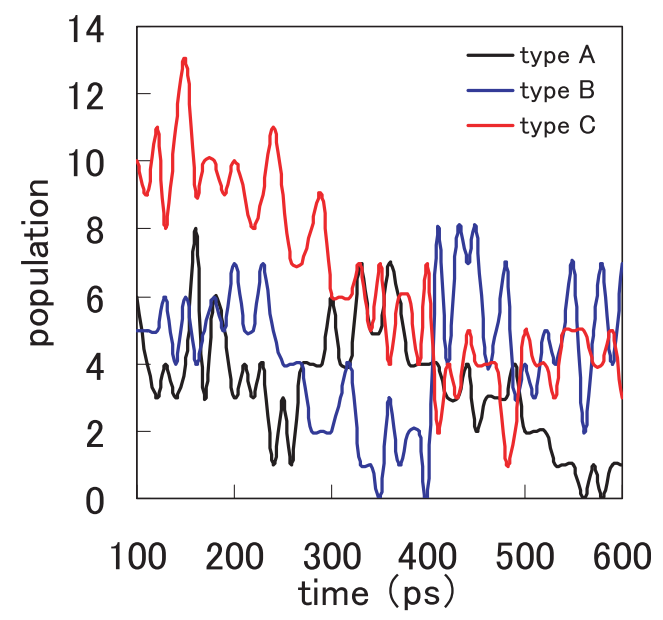

(b) $1 \mathrm{G}+\mathrm{CUR}$

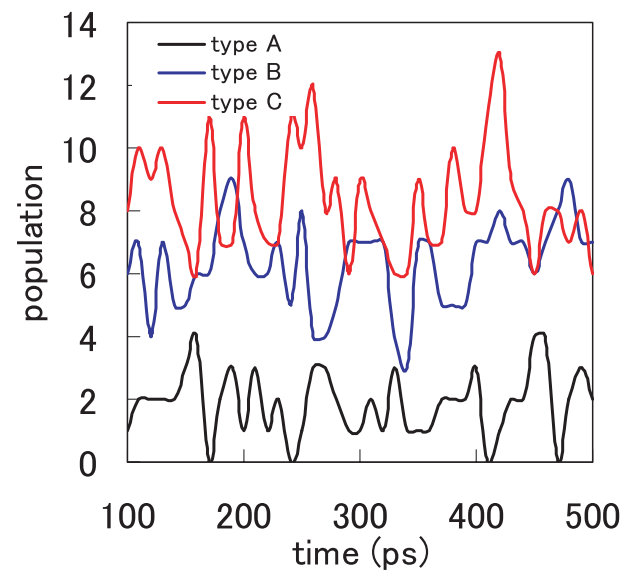

Fig. 7 Fluctuation of the population of the hydrogen bonds in (a) CUR and (b) 1G+CUR. The black, blue and red lines indicate the types A, B, and C hydrogen bonds, respectively.

that of other waters. A snapshot of $1 \mathrm{G}+\mathrm{CUR}$ during the MD is shown in Fig. 9. One or two water molecules existed between the main and the side chains, and hydrogen bonds connect them. These water molecules may be a bound water molecule suggested by Teramoto et al. [7] The main chain was pulled toward the side chain by these hydrogen bonds, which is a reason that the main chain was bent around the side chain.

\section{Conclusion}

This study simulates CUR and 1G+CUR in water by molecular dynamics calculation, to understand how the introduction of the glucose side chain affects the entire helical structure from the molecular level. The calculation showed that the water molecules neighboring the side chain are bound with hydrogen bonds rather tightly and

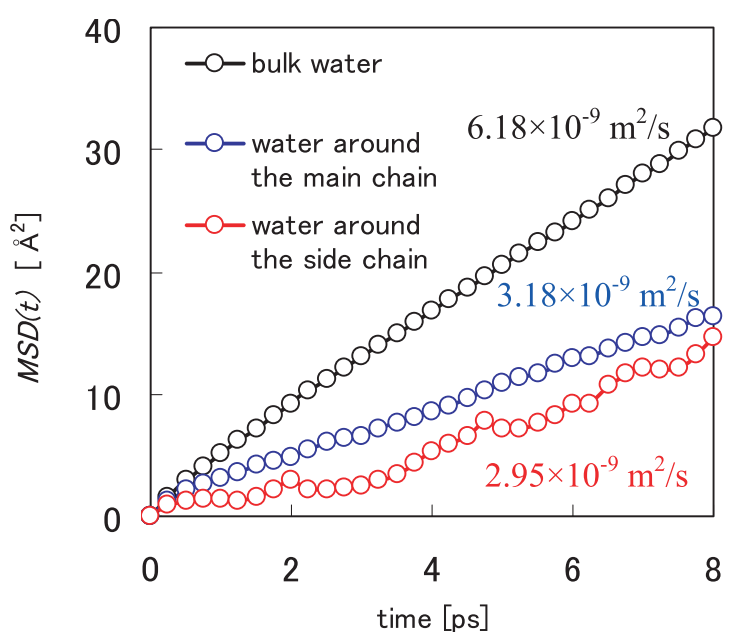

Fig. 8 The mean square displacement for the water molecules for the bulk waters, the waters around the main chain (the water molecules within about $3 \AA$ from the main chain), and the waters around the side chain (the water molecules within about $3 \AA$ from the side chain). The slope corresponds to the self-diffusion coefficients.

the mobility is more reduced than that of other water molecules. These bound water molecules are the reason why the helix pitch of CUR is larger than that of $1 \mathrm{G}+$ CUR.

\section{Experimental}

\section{4-1. Construction of Models}

We carried out MD simulations for two models, CUR and $1 \mathrm{G}+\mathrm{CUR}$. We constructed initial structures from the Miyoshi's curdlan model whose pitch is $17.6 \AA$. [13] Their model used exactly the same atomic coordinates as Deslandes et al.,[4] except for the hydrogen-atomic coordinates in the 2 nd hydroxyl group. Since the glucose ring takes the chair conformation at moderate temperatures, two torsional and one bridge angles around the glycosidic bond essentially determine the polysaccharide conformation. These key parameters are defined by $\phi(\mathrm{H} 1-\mathrm{C} 1-\mathrm{O} 3$ '-C3'), $\phi(\mathrm{C} 1-\mathrm{O} 3$ '-C3'-H3') and $\tau\left(\mathrm{C} 1-\mathrm{O} 3{ }^{\prime}-\mathrm{C} 3{ }^{\prime}\right)$ as illustrated in Fig. 1. According to the crystallographic data of the dehydrated curdlan, we used $\phi=29.1^{\circ}, \phi=9.6^{\circ}$ and $\tau=110.4^{\circ}$ for CUR. [4] We constructed $1 \mathrm{G}+\mathrm{CUR}$ by attaching a side chain to only one main-chain of CUR. All models have 19 glucoses on each chain, thus total 57 main chain glucoses are present. Atomic charges of each model were calculated by single point energy calculation using AM1 method in CAChe Ver 5.0. [14] 


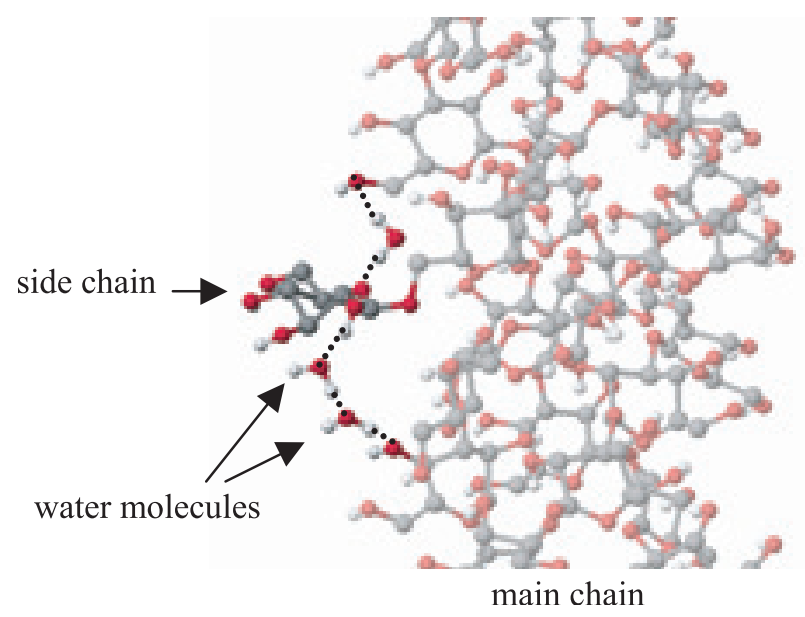

Fig. 9 A snapshot of $1 \mathrm{G}+\mathrm{CUR}$ during the $\mathrm{MD}$ simulation. Dotted lines indicate hydrogen bonds. The main chain is drawn by translucent colors.

\section{4-2. Computational Methods and Assumptions}

MD simulations were carried out for the $\beta-1,3-D-$ glucan models solvated by water using periodic boundary conditions at NTP ensemble by Materials Explorer Ver. 2.0 Ultra (Fujitsu Ltd. Japan).[15] The Cut-off for Coulomb force interaction was $10 \AA$. The $\beta$-1,3-D-glucan models were placed at the center of a periodic box and solvated by 20000 water molecules. The cell was cubic $80 \AA$ on a side. The AMBER force field as shown in Appendix was used for these models throughout this study. Those force field parameters were built by Direct Force Field Ver. 3.0 (Aeon Technology, Inc.). [16] Direct Force Field is an expert system that can be used to build molecular dynamics force fields automatically from ab initio and experimental data.[17-19] For the water molecules, the TIP3P force field was used. The MD system was equilibrated at $298 \mathrm{~K}$ setting the integration time step $(\Delta t)$ to $0.5 \mathrm{fs}$, and the temperature was controlled by the Nose method. The total calculation time was 700 ps. For the other parameters and methods, the default sets in Materials Explorer were used. We also calculated the $M S D(t)$ of the water molecules during 8 ps after MD simulation. We also calculated the same system using GLYCAM94 parameters and the similar results were obtained. We are in the middle of comparing detailed difference between the GLYCAM and our parameter sets.

\section{References}

1. "Biopolymers, Polysaccharides II : Polysaccharides from Eukaryote"s, Vol. 6 (E. J.Vandamme, Ed.) Wiley-VCH, Weinheim, (2002).

2. N. R. Di Luzio, D. L. Williams, R. B. McNamee, B. F. Edwards, A. Kitahara, Int. J. Cancer, 24, 773 (1979).

3. P. P. Jagodzinski, R. Wiaderkiewicz, G. Kurzawski, M. Kloczewiak, H. Nakashima, E. Hyjek, N. Yamamoto, T. Uryu, Y. Kaneko, M. R. Posner, D. Kozbor, Virology, 202, 735 (1994).

4. Y. Deslandes, R. H. Marchessault, A. Sarko, Macromolecules, 13, 1466 (1980).

5. R. H. Marchessault, Y. Deslandes, K. Ogawa, P. R. Sundararajan, Can. J. Chem., 55, 300 (1977).

6. K. Okuyama, A. Otsubo, Y. Fukuzawa, T. Harada, N. Kasai, Carbohydr. Chem., 10, 645 (1911).

7. A. Teramoto, Progress in Poly. Phys., 41, 25 (1998).

8. K. Koumoto, R. Karinaga, M. Mizu, T. Anada, K. Sakurai, T. Kunitake, S. Shinkai, Biopolymers, 75, 403 (2004).

9. E. D. T. Atkins, K. D. Parker, Nature, 220, 784 (1968).

10. T. Norisuye, T. Yanaki, H. Fujita, J. Polym. Sci., Polym. Phys. Ed., 18, 547(1980).

11. B. K.Sathyanarayana, V. S. R. Rao, Biopolymers, 11, 1379 (1972).

12. V. Frecer, R. Rizzo, S. Miertus, Biomacromol., 1, 91 (2000).

13. K. Miyoshi, K. Uezu, K. Sakurai, S. Shinkai, Chem. Biodiv., 1, 916 (2004).

14. CAChe Release 5.0, Serial No. 150270302, Fujitsu ltd. Japan.

15. Materials Explorer Ver 2.0 Ultra, Serial No. 7582972775121002442252-U212, Fujitsu Ltd. Japan.

16. Direct Force Field Release 3. 0, license No. SF02 AE0038, Aeon Technology, Inc.

17. W. Chuanjie, L. Xiaofeng, D. Jianxing, S. Huai, Fluid Phase Equilib. , 236, 66 (2005).

18. D. Jianxing, W. Chuanjie, B. Xiaoguang, S. Huai, Fluid Phase Equilib., 236, 78 (2005).

19. H. Sun, Fluid Phase Equilib., 217, 59 (2004). 


\section{Appendix}

AMBER Force Field parameters

$$
\begin{aligned}
& \sum_{\text {bond }} K_{b}\left(b-b_{0}\right)^{2}+ \\
& \sum_{\text {angle }} K_{\theta}\left(\theta-\theta_{0}\right)^{2}+ \\
& \sum_{\text {dihedral }} \frac{K_{\phi}}{2}\left[1+\cos \left(n \phi-\phi_{0}\right)\right]+ \\
& \sum_{\text {nonbond }}\left\{\varepsilon_{i j}\left[\left(\frac{R_{i j}^{0}}{R_{i j}}\right)^{12}-2\left(\frac{R_{i j}^{0}}{R_{i j}}\right)^{6}\right]+\frac{q_{i} q_{j}}{R_{i j}}\right\} \\
& \varepsilon_{i j}=\sqrt{\varepsilon_{i} \varepsilon_{j}} \\
& R_{i j}^{0}=\frac{R_{i}^{0}+R_{j}^{0}}{2}
\end{aligned}
$$

\begin{tabular}{|c|c|c|c|c|}
\hline \multicolumn{4}{|c|}{ atom type 1 atom type 2 atom type $3 K_{\theta}[\mathrm{kcal} / \mathrm{mol}]$} & \multirow{2}{*}{$\frac{\theta_{0}[\mathrm{deg}]}{110.551}$} \\
\hline $\mathrm{OS}$ & $\mathrm{C} \mathrm{T}$ & $\mathrm{C} \mathrm{T}$ & 71.905 & \\
\hline $\mathrm{OS}$ & $\mathrm{CT}$ & H 1 & 48.346 & 104.915 \\
\hline $\mathrm{C} \mathrm{T}$ & $\mathrm{C} \mathrm{T}$ & H 1 & 37.322 & 106.226 \\
\hline $\mathrm{C} \mathrm{T}$ & $\mathrm{OS}$ & $\mathrm{C} \mathrm{T}$ & 112.491 & 98.163 \\
\hline $\mathrm{C} \mathrm{T}$ & $\mathrm{C} \mathrm{T}$ & $\mathrm{C} \mathrm{T}$ & 110.047 & 76.870 \\
\hline $\mathrm{C} \mathrm{T}$ & $\mathrm{C} \mathrm{T}$ & $\mathrm{H} \mathrm{C}$ & 105.262 & 47.361 \\
\hline $\mathrm{C} \mathrm{T}$ & $\mathrm{C} \mathrm{T}$ & $\mathrm{OH}$ & 62.534 & 108.045 \\
\hline $\mathrm{OH}$ & $\mathrm{C} \mathrm{T}$ & H 1 & 55.186 & 111.666 \\
\hline $\mathrm{C} \mathrm{T}$ & $\mathrm{OH}$ & $\mathrm{HO}$ & 58.125 & 107.602 \\
\hline $\mathrm{OS}$ & $\mathrm{C} \mathrm{T}$ & H 2 & 51.304 & 106.097 \\
\hline $\mathrm{OH}$ & $\mathrm{C} \mathrm{T}$ & $\mathrm{H} 2$ & 56.502 & 105.748 \\
\hline $\mathrm{C} \mathrm{T}$ & $\mathrm{C} \mathrm{T}$ & H 2 & 28.604 & 107.991 \\
\hline $\mathrm{OS}$ & $\mathrm{C} \mathrm{T}$ & $\mathrm{OH}$ & 93.045 & 111.110 \\
\hline H 1 & $\mathrm{C} \mathrm{T}$ & H 1 & 40.850 & 105.901 \\
\hline $\mathrm{O} \mathrm{S}$ & $\mathrm{C} \mathrm{T}$ & $\mathrm{OS}$ & 93.045 & 111.110 \\
\hline
\end{tabular}

\begin{tabular}{|c|c|c|c|c|c|c|}
\hline $\begin{array}{l}\text { atom } \\
\text { type } 1\end{array}$ & $\begin{array}{l}\text { atom } \\
\text { type } 2\end{array}$ & $\begin{array}{l}\text { atom } \\
\text { type } 3\end{array}$ & $\begin{array}{l}\text { atom } \\
\text { type } 4\end{array}$ & $\begin{array}{c}K_{\phi} \\
{[\mathrm{kcal} / \mathrm{mol}]}\end{array}$ & $\begin{array}{c}\phi_{0} \\
{[\mathrm{kcal} / \mathrm{mol}]}\end{array}$ & $\begin{array}{c}n \\
{[\mathrm{kcal} / \mathrm{mol}]}\end{array}$ \\
\hline $\mathrm{CT}$ & $\mathrm{CT}$ & OS & C T & 0.161 & 0.000 & 3.000 \\
\hline H 1 & C T & OS & $\mathrm{C} \mathrm{T}$ & 1.301 & 0.000 & 3.000 \\
\hline O S & $\mathrm{CT}$ & $\mathrm{CT}$ & $\mathrm{C} \mathrm{T}$ & 2.982 & 0.000 & 3.000 \\
\hline O S & C T & $\mathrm{C} \mathrm{T}$ & $\mathrm{HC}$ & 1.871 & 0.000 & 3.000 \\
\hline H 1 & C T & $\mathrm{C} \mathrm{T}$ & $\mathrm{C} \mathrm{T}$ & -0.255 & 0.000 & 3.000 \\
\hline H 1 & C T & $\mathrm{C} \mathrm{T}$ & $\mathrm{HC}$ & -0.265 & 0.000 & 3.000 \\
\hline $\mathrm{CT}$ & $\mathrm{C} \mathrm{T}$ & $\mathrm{C} \mathrm{T}$ & $\mathrm{C} \mathrm{T}$ & 0.478 & 0.000 & 3.000 \\
\hline $\mathrm{C} \mathrm{T}$ & C T & C T & $\mathrm{HC}$ & 0.074 & 0.000 & 3.000 \\
\hline $\mathrm{HC}$ & C T & $\mathrm{C} \mathrm{T}$ & $\mathrm{HC}$ & 0.300 & 0.000 & 3.000 \\
\hline O S & C T & $\mathrm{C} \mathrm{T}$ & O S & 4.904 & 0.000 & 3.000 \\
\hline O S & C T & $\mathrm{C} \mathrm{T}$ & $\mathrm{OH}$ & 4.904 & 0.000 & 3.000 \\
\hline H 1 & $\mathrm{C} \mathrm{T}$ & $\mathrm{C} \mathrm{T}$ & $\mathrm{OH}$ & 1.347 & 0.000 & 3.000 \\
\hline $\mathrm{CT}$ & C T & $\mathrm{C} \mathrm{T}$ & $\mathrm{OH}$ & 2.130 & 0.000 & 3.000 \\
\hline $\mathrm{HC}$ & C T & $\mathrm{C} \mathrm{T}$ & $\mathrm{OH}$ & 1.219 & 0.000 & 3.000 \\
\hline $\mathrm{CT}$ & C T & $\mathrm{OH}$ & HO & 0.082 & 0.000 & 3.000 \\
\hline H 1 & $\mathrm{C} \mathrm{T}$ & $\mathrm{OH}$ & $\mathrm{HO}$ & 0.897 & 0.000 & 3.000 \\
\hline H 1 & C T & $\mathrm{C} \mathrm{T}$ & H 1 & -2.072 & 0.000 & 3.000 \\
\hline $\mathrm{CT}$ & OS & C T & H 2 & -0.193 & 0.000 & 3.000 \\
\hline Н 2 & $\mathrm{C} \mathrm{T}$ & $\mathrm{OH}$ & $\mathrm{HO}$ & 0.819 & 0.000 & 3.000 \\
\hline H 2 & C T & $\mathrm{C} \mathrm{T}$ & $\mathrm{C} \mathrm{T}$ & -3.108 & 0.000 & 3.000 \\
\hline H 2 & $\mathrm{CT}$ & $\mathrm{C} \mathrm{T}$ & $\mathrm{HC}$ & -1.289 & 0.000 & 3.000 \\
\hline $\mathrm{CT}$ & O S & C T & $\mathrm{OH}$ & 1.524 & 0.000 & 3.000 \\
\hline $\mathrm{OH}$ & C T & C T & $\mathrm{OH}$ & 2.254 & 0.000 & 3.000 \\
\hline O S & $\mathrm{C} \mathrm{T}$ & C T & H 1 & -0.698 & 0.000 & 3.000 \\
\hline O S & $\mathrm{C} \mathrm{T}$ & $\mathrm{OH}$ & $\mathrm{HO}$ & 1.005 & 0.000 & 3.000 \\
\hline $\mathrm{C} \mathrm{T}$ & O S & $\mathrm{C} \mathrm{T}$ & O S & 1.524 & 0.000 & 3.000 \\
\hline H 2 & C T & C T & H 1 & -1.289 & 0.000 & 3.000 \\
\hline H 2 & C T & C T & $\mathrm{OH}$ & 1.347 & 0.000 & 3.000 \\
\hline
\end{tabular}

Parameters

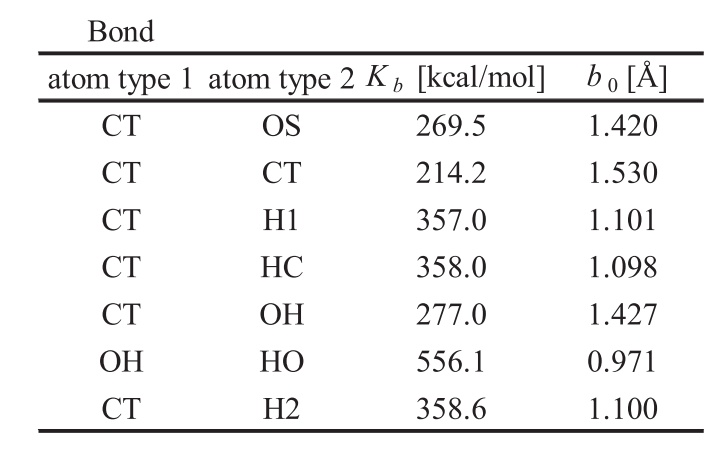

Angle

\begin{tabular}{ccc} 
nonbond & & \\
\hline atom type 1 & $\varepsilon_{\mathrm{n}}^{0}[\mathrm{kcal} / \mathrm{mol}]$ & $R_{\mathrm{n}}^{0}[\AA]$ \\
\hline C T & 0.064 & 3.767 \\
$\mathrm{O} \mathrm{S}$ & 0.088 & 3.234 \\
$\mathrm{H} 1$ & 0.025 & 2.820 \\
$\mathrm{H} \mathrm{C}$ & 0.025 & 2.820 \\
$\mathrm{O} \mathrm{H}$ & 0.088 & 3.234 \\
$\mathrm{H} \mathrm{O}$ & 0.010 & 1.000 \\
$\mathrm{H} 2$ & 0.025 & 2.820 \\
\hline
\end{tabular}

